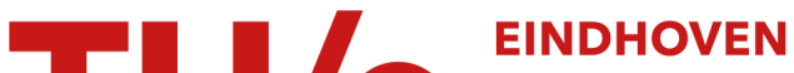 \\ UNIVERSITY OF \\ TECHNOLOGY
}

\section{Quantum Mechanical Repulsion of Exciton Levels in a Disordered Quantum Well}

\section{Citation for published version (APA):}

Intonti, F., Emiliani, V., Lienau, C., Elsaesser, T., Savona, V., Runge, E., Zimmermann, R., Nötzel, R., \& Ploog, K. (2001). Quantum Mechanical Repulsion of Exciton Levels in a Disordered Quantum Well. Physical Review Letters, 87(7), 076801-1/4. [076801]. https://doi.org/10.1103/PhysRevLett.87.076801

DOI:

10.1103/PhysRevLett.87.076801

Document status and date:

Published: 01/01/2001

\section{Document Version:}

Publisher's PDF, also known as Version of Record (includes final page, issue and volume numbers)

\section{Please check the document version of this publication:}

- A submitted manuscript is the version of the article upon submission and before peer-review. There can be important differences between the submitted version and the official published version of record. People interested in the research are advised to contact the author for the final version of the publication, or visit the $\mathrm{DOI}$ to the publisher's website.

- The final author version and the galley proof are versions of the publication after peer review.

- The final published version features the final layout of the paper including the volume, issue and page numbers.

Link to publication

\section{General rights}

Copyright and moral rights for the publications made accessible in the public portal are retained by the authors and/or other copyright owners and it is a condition of accessing publications that users recognise and abide by the legal requirements associated with these rights.

- Users may download and print one copy of any publication from the public portal for the purpose of private study or research.

- You may not further distribute the material or use it for any profit-making activity or commercial gain

- You may freely distribute the URL identifying the publication in the public portal.

If the publication is distributed under the terms of Article 25fa of the Dutch Copyright Act, indicated by the "Taverne" license above, please follow below link for the End User Agreement:

www.tue.nl/taverne

Take down policy

If you believe that this document breaches copyright please contact us at:

openaccess@tue.nl

providing details and we will investigate your claim. 


\title{
Quantum Mechanical Repulsion of Exciton Levels in a Disordered Quantum Well
}

\author{
Francesca Intonti, Valentina Emiliani,* Christoph Lienau, and Thomas Elsaesser \\ Max-Born-Institut für Nichtlineare Optik und Kurzzeitspektroskopie, Max-Born-Strasse 2A, D-12489 Berlin, Germany \\ Vincenzo Savona, Erich Runge, and Roland Zimmermann \\ Institut für Physik, Humboldt-Universität zu Berlin, Hausvogteiplatz 5-7, D-10117 Berlin, Germany \\ Richard Nötzel and Klaus H. Ploog \\ Paul-Drude-Institut für Festkörperelektronik, Hausvogteiplatz 5-7, D-10117 Berlin, Germany
}

(Received 15 January 2001; published 25 July 2001)

\begin{abstract}
Spatially resolved photoluminescence spectra of a single quantum well are recorded by near-field spectroscopy. A set of over four hundred spectra displaying sharp emission lines from localized excitons is subject to a statistical analysis of the two-energy autocorrelation function. An accurate comparison with a quantum theory of the exciton center-of-mass motion in a two-dimensional spatially correlated disordered potential reveals clear signatures of quantum mechanical energy level repulsion, giving the spatial and energetic correlations of excitons in disordered quantum systems.
\end{abstract}

DOI: 10.1103/PhysRevLett.87.076801

Localized states play a key role for the optical and transport properties of disordered quantum systems. Inhomogeneous broadening of optical spectra is quantum mechanically understood in terms of wave function localization, i.e., the broken translational symmetry leads to slightly different eigenenergies and, thus, transition energies. For low-dimensional semiconductor structures, quantum wells and quantum wires, this scenario has received direct convincing support by spatially resolved exciton spectroscopy on a $100 \mathrm{~nm}$ scale, using either near-field optical spectroscopy [1-3] or microphotoluminescence ( $\mu \mathrm{PL})$ [4]. These techniques allow one to resolve the smooth inhomogeneously broadened line of macroscopic PL spectra into many narrow excitonic spikes whose individual spectral widths are often determined by experimental resolution or by the natural linewidth.

Among the key concepts in the quantum theory of localization and the related "weak localization" effects are the spatial and energetic correlations of wave functions as well as the corresponding correlations of energy levels [5]. In disorder-induced metal-insulator (Anderson) transitions, in the insulating phase, two wave functions having almost identical energies will, in general, be localized in different spatial areas. In the conducting phase, wave functions always show a non-negligible spatial overlap. This is reflected in a level repulsion of the eigenenergies, which can be visualized as splitting of "bonding and antibonding" combinations of (many) quasidegenerate states. Quantum mechanical level repulsion in complex and disordered quantum systems has first been modeled by Wigner and Dyson's random matrix theory. Its predictions for the level-level correlation function saw some impressive experimental verifications in various branches of physics over the past 70 years [6] and became a most valuable criterion for the theoretical study of the Anderson transition $[7,8]$.
PACS numbers: 73.20.Fz, 07.79.Fc, 78.55.Cr, 78.67.De

Excitons in quantum wells and wires are localized on a nanometer scale due to disorder. Thus, level repulsion contributes only weakly to macroscopic spectra, while spatially resolved spectroscopy, exciting only wave functions which are close to each other in real space, should give direct insight into this typical quantum mechanical hallmark of localization.

In this Letter, we present the first clear evidence and analysis for the repulsion of exciton levels by combining near-field photoluminescence spectroscopy of a single quantum well with a quantitative theoretical modeling in terms of disorder eigenstates of a random potential. We demonstrate that, for small energy separations $\Delta E$ of eigenstates, the two-energy autocorrelation function is strongly influenced by level repulsion, whereas correlations of the potential energy itself influence the energy-level correlations at larger $\Delta E$.

As a model system, we investigate a $\mathrm{GaAs} / \mathrm{AlGaAs}$ quantum well sample grown by molecular beam epitaxy on a GaAs (311)A substrate [9]. It consists of a single $3 \mathrm{~nm}$ thick GaAs quantum well layer clad between two $50 \mathrm{~nm}$ thick $\mathrm{Al}_{0.5} \mathrm{Ga}_{0.5} \mathrm{As}$ barrier layers and capped with $20 \mathrm{~nm}$ of GaAs [Fig. 1(a)]. A low-temperature near-field scanning optical microscope [10] is used in an illumination/collection geometry with a combined spatial and spectral resolution of $150 \mathrm{~nm}$ and $100 \mu \mathrm{eV}$, respectively. In this geometry, the sample is excited with light from a HeNe laser $(1.96 \mathrm{eV})$, transmitted through a chemically etched, uncoated near-field fiber probe [11]. Photoluminescence from the sample is collected through the same probe, dispersed in a $f=50 \mathrm{~cm}$ monochromator, and detected with a cooled charge coupled device.

The averaged photoluminescence spectrum of the sample [Fig. 1(b)] shows a $15 \mathrm{meV}$ broad emission band. This spectrum is obtained by averaging over 432 individual near-field spectra recorded by scanning a $1.2 \times 3.6 \mu \mathrm{m}^{2}$ 

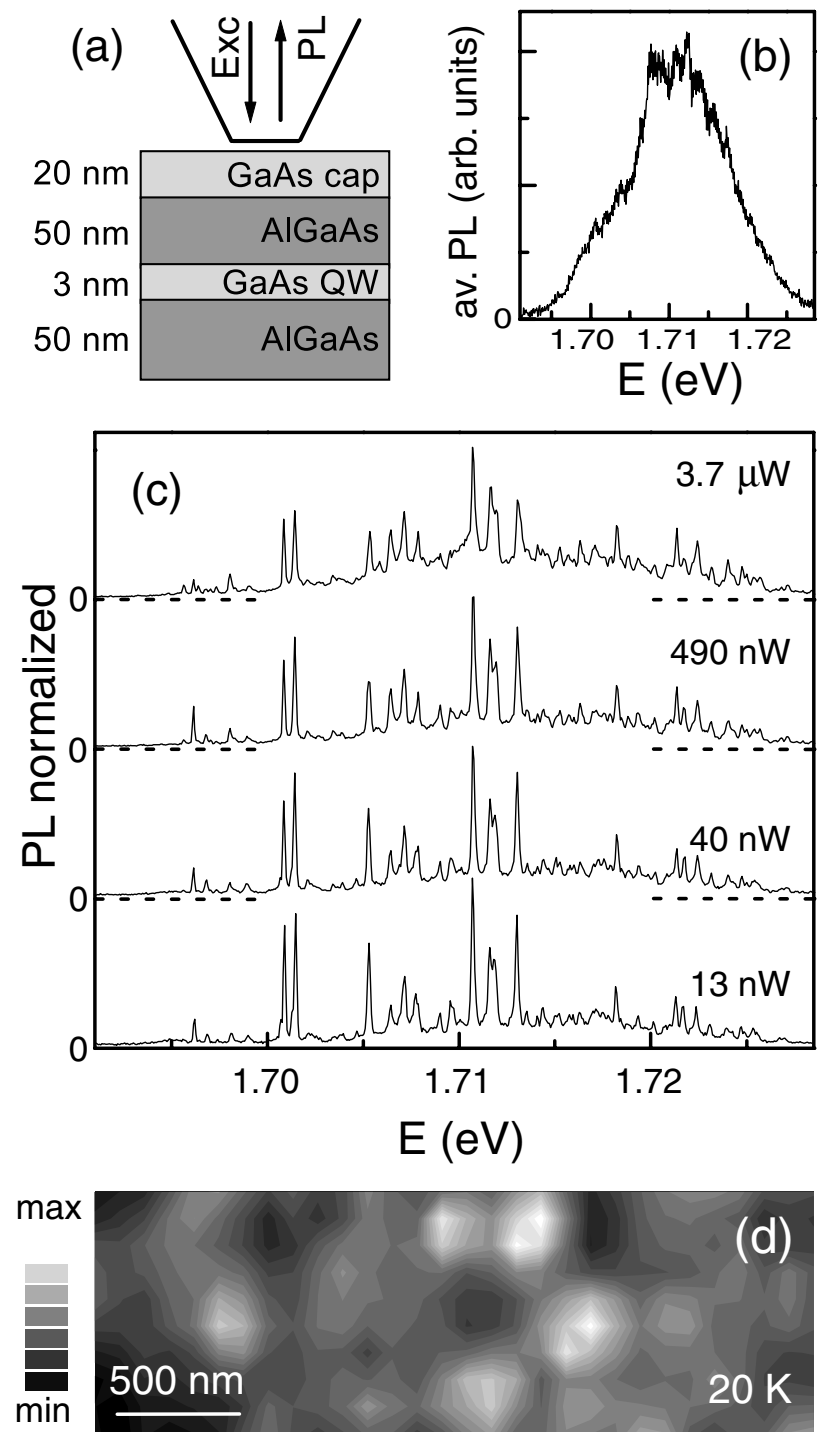

FIG. 1. (a) Schematic experimental setup. (b) Averaged PL spectrum $(T=20 \mathrm{~K})$, excitation power $800 \mathrm{nW}$. (c) Representative near-field PL spectra at a fixed spatial position for excitation intensities varied over about 3 orders of magnitude. (d) Two-dimensional $\left(1 \times 3 \mu \mathrm{m}^{2}\right)$ spectrally averaged PL image.

region with steps of $100 \mathrm{~nm}$. Such sets of near-field spectra are recorded at a temperature $T=20 \mathrm{~K}$ for different excitation powers between $100 \mathrm{nW}$ and $4 \mu \mathrm{W}$ coupled into the fiber probe. In contrast to the averaged emission, the individual near-field spectra break up into a set of spectrally sharp and intense emission lines [1,2]. Representative near-field PL spectra recorded at a fixed spatial position are displayed in Fig. 1(c) for excitation powers between $10 \mathrm{nW}$ and $4 \mu \mathrm{W}$. The spatial extension of the individual spikes is limited by our spatial resolution of $150 \mathrm{~nm}$ [12]. The linear intensity dependence and excitation independent shape of the emission spectra rule out biexcitonic transitions [4] and make contributions from charged excitons [13] unlikely.
The spectrally sharp emission spikes originate from excitons localized within the quantum well disorder potential caused by interface roughness and alloy fluctuations. Provided the resulting local energy fluctuations are not larger than the exciton binding energy, the relative electron-hole motion can be assumed as undistorted by disorder [14]. In this case, the center-of-mass (COM) motion of the exciton obeys the Schrödinger equation with an effective disordered potential $V(\mathbf{R})$. It results from a convolution of the microscopic confinement energy profile with the exciton $1 s$-wave function of exponential shape $[14,15]$. In our numerical calculations, we take the confinement energy profile $U(\mathbf{R})$ as random and spatially uncorrelated (on an atomic length scale). Realizations of the effective potential are generated via $V(\mathbf{R}) \propto \int \exp \left(-\left|\mathbf{R}-\mathbf{R}^{\prime}\right| / \xi\right) U\left(\mathbf{R}^{\prime}\right) d \mathbf{R}^{\prime}$. The quantity $\xi$ determines the spatial correlation length of the potential, while the average potential amplitude is given by $\sigma^{2}=\left\langle V^{2}(0)\right\rangle$. As $\xi$ is related both to the exciton Bohr radius and to the microscopic structure of the interfaces, it should be taken as an adjustable parameter in the present context. Any ensemble-average property of the Schrödinger equation depends only on the ratio $\sigma / E_{c}$, where $E_{c}=\hbar^{2} /\left(2 M \xi^{2}\right)$ is a natural energy unit for the COM confinement ( $M$ : exciton mass) [16].

Neglecting polaritonic effects, the optical strength of a given excitonic COM eigenstate $\alpha$ is proportional to the spatially integrated COM wave function, $M_{\alpha}=$ $\int \psi_{\alpha}(\mathbf{R}) d \mathbf{R}$. This allows one to express the optical density of a single spot $n$ as

$$
D_{n}(E)=\frac{1}{A} \sum_{\alpha} M_{\alpha, n}^{2} \delta\left(E-\epsilon_{\alpha, n}\right),
$$

where $\epsilon_{\alpha, n}$ are the energy eigenvalues and $A$ is the spot area. Equation (1) is proportional to the local absorption spectrum and directly related to the experimentally measured local PL. A detailed description of PL spectra requires the introduction of population factors $N_{\alpha, n}$ in Eq. (1) which can be obtained only from a microscopic simulation of the relaxation dynamics [14,17], being beyond the scope of this Letter.

The optical density - either from a single microscopic spot or spatially averaged - does not bear much information on the nature of the underlying disordered system [17]. Detailed information can, however, be obtained from two-point energy correlation functions. We define the optically weighted autoconvolution of the spectrum,

$$
\begin{aligned}
R(\Delta E) & =A\left\langle\int d E^{\prime} D_{n}\left(E^{\prime}\right) D_{n}\left(E^{\prime}-\Delta E\right)\right\rangle \\
& =\frac{1}{A}\left\langle\sum_{\alpha, \beta} M_{\alpha, n}^{2} M_{\beta, n}^{2} \delta\left[\Delta E-\left(\epsilon_{\alpha, n}-\epsilon_{\beta, n}\right)\right]\right\rangle .
\end{aligned}
$$

Hereafter, $\langle\cdots\rangle$ denotes the average over the measured spots in the experiment or over an ensemble of disorder 
realizations in the simulation. The quantity $R(\Delta E)$ represents the average distribution of energy-level distances weighted by the optical strengths. The corresponding uncorrelated quantity is defined as

$$
R_{0}(\Delta E)=A \int d E^{\prime}\left\langle D_{n}\left(E^{\prime}\right)\right\rangle\left\langle D_{n}\left(E^{\prime}-\Delta E\right)\right\rangle .
$$

As defined, both $R(\Delta E)$ and $R_{0}(\Delta E)$ are extensive quantities. They are dominated by the contribution of pairs of localized eigenstates which are well separated in space and therefore not correlated. An intensive quantity is the correlated part of the autoconvolution,

$$
R_{c}(\Delta E)=R(\Delta E)-R_{0}(\Delta E) .
$$

In this quantity, the contribution from pairs of uncorrelated levels cancels and only those pairs of states whose relative distance is below their localization length contribute. Thus, $R_{c}(\Delta E)$, called autocorrelation, represents a microscopic statistical property of the system and, contrary to the optical density, depends dramatically on the parameter $\sigma / E_{c}$. The same level autocorrelation function manifests itself in the time domain as a faint oscillation in the time-resolved coherent emission (resonant Rayleigh scattering) [16].

The concept of level repulsion is illustrated in Fig. 2(a). Wave functions $\alpha$ and $\beta$ are well separated in real space
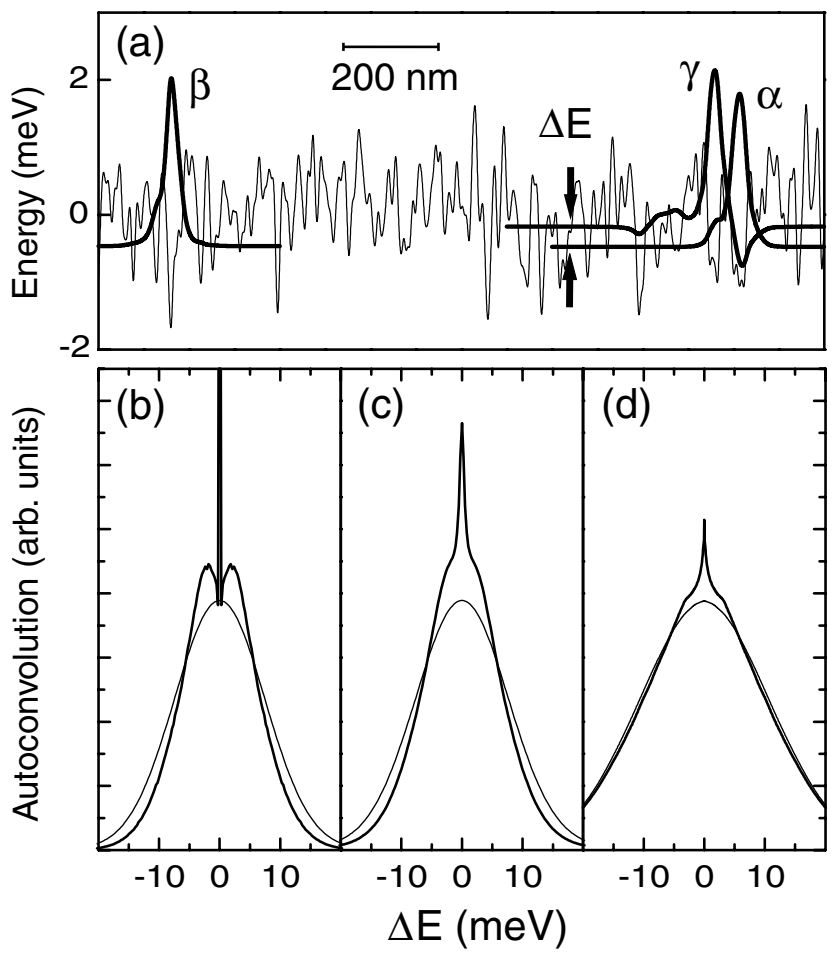

FIG. 2. (a) Schematic illustration of the level repulsion effect. Spatially overlapping localized wave functions are energetically nondegenerate. (b) - (d) Averaged spectral autoconvolution $R(\Delta E)$ (solid line) and the uncorrelated part $R_{0}(\Delta E)$ (thin line). (b) Numerical simulation. (c) Numerical simulation convoluted with a Lorentzian. (d) Experiment at $20 \mathrm{~K}$ and an excitation power of $800 \mathrm{nW}$. and may thus be nearly degenerate. On the contrary, the localized wave functions $\alpha$ and $\gamma$ are spatially overlapping and therefore energetically well separated. The quantum mechanical correlations have to be distinguished from those correlations present in the classical limit, $E_{c} / \sigma \rightarrow 0$ [16]. Here, the kinetic energy term is negligible and the eigenenergies are simply the values assumed by $V(\mathbf{R})$. Thus, states that are close in real space have similar energies, resulting in positive values of $R_{c}(\Delta E)$ as $\Delta E \rightarrow 0$. As $\int R_{c}(\Delta E) d \Delta E$ vanishes by definition, $R_{c}(\Delta E)<0$ at large $\Delta E$.

Our numerical simulations were performed on a grid of $32 \times 32$ points corresponding to a square region of $135 \mathrm{~nm}$, which represents the measured spot. We numerically computed the eigenvalues and eigenfunctions of the Schrödinger equation with periodic boundary conditions for $10^{3}$ realizations of $V(\mathbf{R})$. We then evaluated the quantities (3), (4), and (5) by performing ensemble averages over the numerical data. The same quantities have been computed from the experimental data. In contrast to the optical density (1), the integrated weight of the measured PL spectra varies with position [Fig. 1(d)]. Possible sources for this nonhomogeneity are systematic trends across the sample, exciton diffusion out of the spot, nonradiative trapping along the relaxation path, and local variations of the excitation efficiency. It can be shown within a kinetic model that the autocorrelation obtained from the normalized experimental spectra is the one to be compared with the numerically computed $R_{c}(\Delta E)$. For this to hold, the fluctuations in the integrated intensity have to be assumed uncorrelated to the shape of the actual microscopic spectra. The same analysis provides a criterion to check this assumption, which is verified for our data. The parameters $\sigma$ and $\xi$ entering the disorder potential are obtained by fitting the computed $R_{c}(\Delta E)$ to the experimental data. To account for the finite experimental linewidth, $R_{c}$ is convoluted with a Lorentzian of width $2 \gamma$ [18]. We obtain from the fit $\sigma=(5.3 \pm 0.2) \mathrm{meV}$ and $\xi=\left(17_{-3}^{+8}\right) \mathrm{nm}$, corresponding to $\sigma / E_{c}=8$ (with $M=0.25 m_{e}$ ). The broadening $\gamma=(0.22 \pm 0.02) \mathrm{meV}$ is consistent with the linewidth measured on the single spikes.

Figures 2(b)-2(d) show the computed and the experimental functions $R(\Delta E)$ and $R_{0}(\Delta E)$. Panel 2(b) displays the curves directly computed from the solutions of the Schrödinger equation. The sharp peak at $\Delta E=0$ involves a single energy grid point and is the numerical counterpart of the Dirac delta contribution $\delta(\Delta E)$ given by the diagonal terms $\alpha=\beta$ in Eq. (3). In panel 2(c), the same curves are convoluted with a Lorentzian of width $2 \gamma$. Panel 2(d) shows the corresponding experimental quantities, recorded at $T=20 \mathrm{~K}$ with an excitation power of $800 \mathrm{nW}$.

Figure 3 compares the computed and the experimental functions $R_{c}(\Delta E)$. The curve calculated without Lorentz convolution (dashed line) displays a pronounced dip for small $\Delta E$ values, the signature of quantum mechanical level repulsion. The arrow in the plot denotes the 


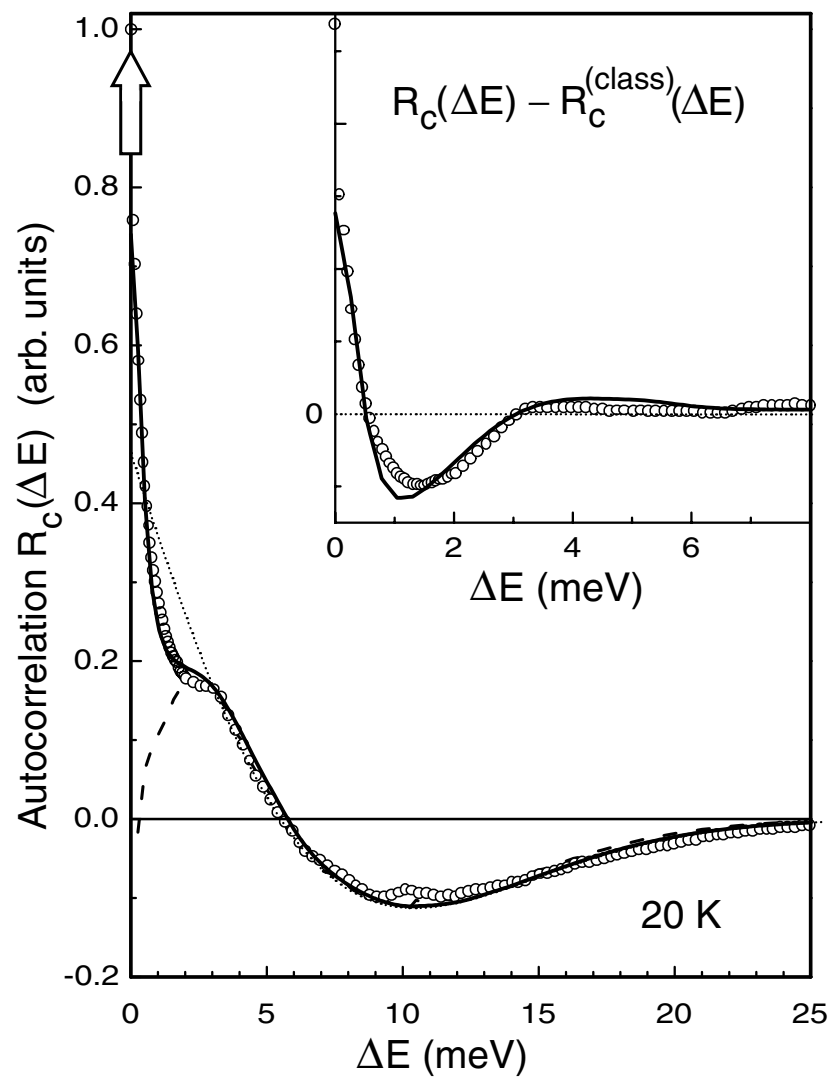

FIG. 3. Autocorrelation function $R_{c}(\Delta E)$ at $T=20 \mathrm{~K}$, shown for the experimental data (circles), the Lorentz-convoluted numerical simulation (solid line), the classical limit (dotted line), and the raw numerical simulation (dashed line). Level repulsion is evident from the shoulder around $3 \mathrm{meV}$ (circles, solid line). The arrow on the vertical axis denotes the $\delta$-function part of the dashed curve. Inset: Difference between $R_{c}(\Delta E)$ and classical autocorrelation $R_{c}^{\text {(class) }}(\Delta E)$. The experimental data are shown as circles and the numerical result as a solid line.

additional delta contribution $\delta(\Delta E)$. The Lorentz convolution gives rise to a broadened peak centered at $\Delta E=0$ and weakens the level repulsion dip into a shoulder (full line). This behavior is in excellent agreement with the experimental result (circles) and strikingly different from the classical limit (dotted line) which neglects level repulsion and is determined solely by the energy correlation of the potential itself. At larger $\Delta E$ values, both calculated and experimental $R_{c}(\Delta E)$ follow the classical autocorrelation. The pronounced level repulsion dip is emphasized in the inset of Fig. 3, showing the difference between our results and the classical limit. The experimental autocorrelations remain essentially unchanged for excitation powers ranging from $100 \mathrm{nW}$ to $4 \mu \mathrm{W}$ and for temperatures between 5 and $20 \mathrm{~K}$, demonstrating that level repulsion of single excitons gives rise to the pronounced shoulder in $R_{c}(\Delta E)$ [19].

In conclusion, the results presented here provide the first clear evidence for exciton level repulsion in a disordered quantum well, opening the way to a systematic approach for the characterization of disordered nanostruc- tures by means of correlation techniques. In the studied disorder potential with a finite spatial correlation length of $\xi=17 \mathrm{~nm}$, the two-energy autocorrelation is determined both by quantum mechanical level repulsion, effective at small $\Delta E$, and by the classical potential energy correlation dominating at large $\Delta E$. In the so-called white noise limit of very short correlation length [16], the level autocorrelation takes negative values over all the $\Delta E$ range, since the quantum mechanical level repulsion is dominant. It appears attractive to explore these different regimes and to compare near-field and far-field, e.g., Rayleigh scattering, experiments to access dynamic localization effects.

This work was supported by the Deutsche Forschungsgemeinschaft (SFB 296) and the European Union through the EFRE and SQID programs and the Marie-Curie Fellowships ERB40001GT975127 and MCFI-1999-00728 for V.E. and V.S., respectively.

*Present address: LENS (Laboratorio Europeo di Spettroscopie non Lineari), I-50125 Firenze, Italy.

[1] H. F. Hess et al., Science 264, 1740 (1994).

[2] D. Gammon et al., Phys. Rev. Lett. 76, 3005 (1996).

[3] G. von Freymann et al., Appl. Phys. Lett. 77, 394 (2000).

[4] K. Brunner et al., Phys. Rev. Lett. 73, 1138 (1994).

[5] I. M. Lifshitz, S. A. Gredeskul, and L. A. Pastur, Introduction to the Theory of Disordered Systems (Wiley, New York, 1988).

[6] M.L. Mehta, Random Matrices (Academic Press, San Diego, 1990), 2nd ed.

[7] B. Kramer and A. MacKinnon, Rep. Prog. Phys. 56, 1469 (1993).

[8] M. Schreiber, in Computational Physics, edited by K. H. Hoffmann and M. Schreiber (Springer, Berlin, 1996).

[9] R. Nötzel et al., Jpn. J. Appl. Phys. 35, L297 (1996).

[10] A. Richter et al., Phys. Rev. Lett. 79, 2145 (1997).

[11] V. Emiliani et al., Phys. Rev. B 61, R10 583 (2000).

[12] F. Intonti et al., Phys. Rev. B 63, 075313 (2001).

[13] A. Hartmann et al., Phys. Rev. Lett. 84, 5648 (2000).

[14] R. Zimmermann, F. Große, and E. Runge, Pure Appl. Chem. 69, 1179 (1997).

[15] H. Castella and J. W. Wilkins, Phys. Rev. B 58, 16186 (1998).

[16] V. Savona and R. Zimmermann, Phys. Rev. B 60, 4928 (1999).

[17] E. Runge and R. Zimmermann, Adv. Solid State Phys. 38, 251 (1998).

[18] A three-step fitting procedure was used: (i) $\sigma$ is independently obtained from the large-energy range of the classical $R_{c}^{\text {(class) }}(\Delta E)$; (ii) numerical simulations were performed for different $\xi$ selecting the value that best fits energy position and amplitude of the level repulsion dip; (iii) $\gamma$ is adjusted by least squares fitting.

[19] Autocorrelation functions of $\mathrm{CdSe}$ quantum wells have been reported in Ref. [3], showing a distinct peak around $18 \mathrm{meV}$. This feature could, in principle, be related to level repulsion. 